\title{
THE MINIMUM NORM OF CERTAIN COMPLETELY POSITIVE MAPS
}

\author{
CHING-YUN SUEN
}

(Communicated by Palle E. T. Jorgensen)

ABstract. Let $L$ be a completely bounded linear map from a unital $C^{*}$ algebra to the algebra of all bounded linear operators on a Hilbert space. Then $\min \left\{\|\phi\|_{\mathrm{cb}}:\left(\begin{array}{cccccc}\phi & & L & 0 & \cdots & 0 \\ & & & & & \vdots \\ L^{*} & & \phi & L & & 0 \\ 0 & & & \ddots & & \\ \vdots & & & & & L \\ 0 & \cdots & 0 & & L^{*} & \phi\end{array}\right)_{n \times n} \begin{array}{l}\text { is completely } \\ \text { positive } \\ \text { for all } n\end{array}\right\}=2 S(L)$, where $S(L)=\min \left\{\|\phi\|_{\mathrm{cb}}: \phi \pm \operatorname{Re} \lambda L\right.$ is completely positive for all $\left.|\lambda|=1\right\}$.

\section{INTRODUCTION}

Let $M_{n}$ denote the $C^{*}$-algebra of complex $n \times n$ matrices generated by the matrix units $E_{i j}(i, j=1,2,3, \ldots, n)$ and $B(H)$ the algebra of all bounded linear operators on a Hilbert space $H$. Let $A$ and $B$ be $C^{*}$-algebras, and let $L: A \rightarrow B$ be a bounded linear map; the map $L$ is called completely positive if $L \otimes I_{n}: A \otimes M_{n} \rightarrow B \otimes M_{n}$ defined by $L \otimes I_{n}(a \otimes b)=L(a) \otimes b$ is positive for all $n$. $L$ is completely bounded if $\sup _{n}\left\|L \otimes I_{n}\right\|$ is finite, and we write $\|L\|_{\mathrm{cb}}=\sup _{n}\left\|L \otimes I_{n}\right\|$. We define $L^{*}(a)=L\left(a^{*}\right)^{*}$ and $S^{\prime}$ the commutant of $S$ contained in $B(H)$. Let $T$ be a bounded linear operators on $H$; the numerical radius of $T$ is $w(T)=\sup _{\|h\|=1}\{|\langle T h, h\rangle|\}$.

In [3, Theorem 2.2], it has been shown that every completely bounded map $L$ from a unital $C^{*}$-algebra $A$ into $B(H)$ has a minimal commutant representation $L(a)=V^{*} T \Pi(a) V$. In [4, Theorem 2.7], we have

$$
\begin{aligned}
S(L) & =\min \left\{\|\phi\|_{\mathrm{cb}}: \phi \pm \operatorname{Re} \lambda L \text { is completely positive for all }|\lambda|=1\right\} \\
& =\min \left\{w(T): L \text { has a minimal commutant representation } V^{*} T \Pi V\right\} .
\end{aligned}
$$

The work in this paper is to sharpen the estimate in the above mentioned theorems. Example 2.8 shows that there exists some case such that

$$
S(L)<\|L\|_{\mathrm{cb}}<\min \left\{\|\phi\|_{\mathrm{cb}}:\left(\begin{array}{cc}
\phi & L \\
L^{*} & \phi
\end{array}\right) \text { is completely positive }\right\}<2 S(L) \text {. }
$$

Received by the editors March 5, 1993 and, in revised form, April 12, 1993.

1991 Mathematics Subject Classification. Primary 46L05.

Key words and phrases. Completely positive map, completely bounded map, numerical radius. 
Hence, if

$$
\left(\begin{array}{cccccc}
\phi & & L & 0 & \ldots & 0 \\
& & & & & \vdots \\
L^{*} & & \phi & L & & 0 \\
0 & & & \ddots & & \\
\vdots & & & & & L \\
0 & \ldots & 0 & & L^{*} & \phi
\end{array}\right)_{n \times n}
$$

is completely positive for all $n$, then $\left(\begin{array}{cc}\phi & L \\ L^{*} & \phi\end{array}\right)$ is completely positive. However, the converse is not true. If $\left(\begin{array}{c}\phi \\ L^{*}\end{array}\right)$ is completely positive, then $\phi \pm \lambda L$ is completely positive for all $|\lambda|=1$. The converse is also not true.

2. THE NUMERICAL RADIUS

Applying the results from Paulsen [2], we have the following properties.

Proposition 2.1. The following three statements are equivalent:

(1) $w(T) \leq 1$.

(2)

$$
\left(\begin{array}{cccccc}
2 & & T & T^{2} & \ldots & T^{n} \\
& & & & & \vdots \\
T^{*} & & 2 & T & & \\
T^{2} & & & \ddots & & T \\
T^{n^{*}} & \ldots & & T^{*} & & 2
\end{array}\right) \geq 0 \text { for all } n .
$$

(3)

$$
\left(\begin{array}{cccccc}
2 & & T & 0 & \ldots & 0 \\
T^{*} & & 2 & T & & 0 \\
0 & & & \ddots & & \\
\vdots & & & & & T \\
0 & \ldots & 0 & & T^{*} & 2
\end{array}\right)_{n \times n} \geq 0 \text { for all } n .
$$

Proof. Using the results from Paulsen [2, p. 36], (1) implies (2).

Suppose that (2) is true. Let

$$
R=\left(\begin{array}{cccccc}
0 & & T & 0 & \ldots & 0 \\
& & & & & \vdots \\
0 & & 0 & T & & 0 \\
0 & & 0 & 0 & & \\
\vdots & & & & \ddots & T \\
0 & \ldots & 0 & 0 & & 0
\end{array}\right),
$$

then

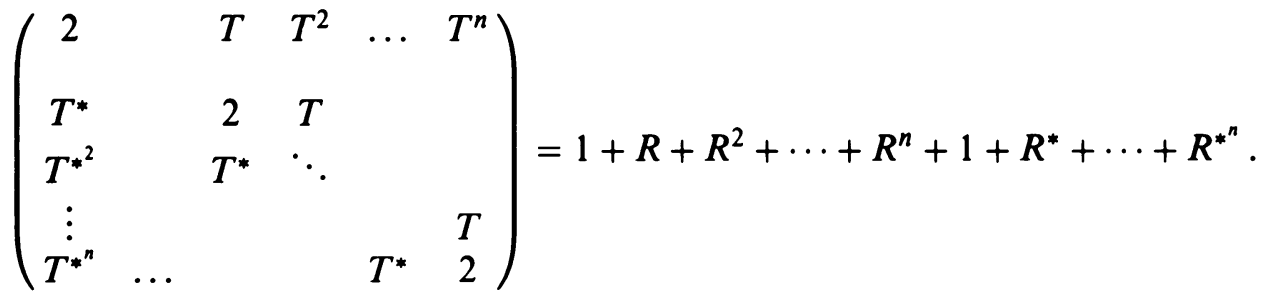


Since

$$
\begin{aligned}
& \left\langle\left(1+R+R^{2}+\cdots+R^{n}+1+R^{*}+\cdots+R^{*^{n}}\right)(1-R) y,(1-R) y\right\rangle \\
& =\left\langle\left(2-R-R^{*}\right) y, y\right\rangle \\
& =\left\langle\left(\begin{array}{cccccc}
2 & & -T & 0 & \ldots & 0 \\
& & & & & \vdots \\
-T^{*} & & 2 & & & 0 \\
0 & & & \ddots & & \\
\vdots & & & & & -T \\
0 & \ldots & 0 & & -T^{*} & 2
\end{array}\right) y, y\right\rangle \geq 0
\end{aligned}
$$

for all $y$ in $H^{n+1}$ and for all $n$, we have (3).

If (3) is true, we claim that $w(T) \leq 1 / \cos \left(\frac{\pi}{n+1}\right)$ for all $n$.

Let

$$
P_{n}^{c}=\left(\begin{array}{cccccc}
2 & & c T & 0 & \ldots & 0 \\
& & & & & \vdots \\
\bar{c} T^{*} & & 2 & c T & & 0 \\
0 & & & 2 & & \\
\vdots & & & & \ddots & c T \\
0 & \ldots & 0 & & \bar{c} T^{*} & 2
\end{array}\right)_{n \times n} ;
$$

then

$$
\begin{aligned}
& P_{n}^{c}=\left(\begin{array}{cccccc}
1 & & 0 & 0 & \ldots & 0 \\
& & & & & \vdots \\
0 & & \bar{c} & 0 & & 0 \\
0 & & 0 & \bar{c}^{2} & & 0 \\
\vdots & & & & \ddots & \\
0 & \ldots & 0 & 0 & & \bar{c}^{n}
\end{array}\right)\left(\begin{array}{cccccc}
2 & & T & 0 & \ldots & 0 \\
& & & & & \vdots \\
T^{*} & & 2 & T & & 0 \\
0 & & T^{*} & 2 & & \\
\vdots & & & & \ddots & T \\
0 & \ldots & 0 & & T^{*} & 2
\end{array}\right) \\
& \times\left(\begin{array}{cccccc}
1 & & 0 & 0 & \ldots & 0 \\
& & & & & \vdots \\
0 & & c & 0 & & 0 \\
0 & & 0 & c^{2} & & 0 \\
\vdots & & & & \ddots & \\
0 & \ldots & 0 & 0 & & c^{n}
\end{array}\right) \geq 0
\end{aligned}
$$

for $|c|=1$ and for all $n$. Let $m$ be a fixed number,

$$
\begin{aligned}
& \lambda=\left(\begin{array}{c}
\lambda_{1} \\
\lambda_{2} \\
\vdots \\
\lambda_{m+1}
\end{array}\right) \quad \text { with }\|\lambda\|=1, \\
& S_{m+1}=\left(\begin{array}{cccccc}
0 & & 1 & 0 & \ldots & 0 \\
& & & & & \vdots \\
0 & & 0 & 1 & & 0 \\
0 & & 0 & 0 & & \\
\vdots & & & & \ddots & 1 \\
0 & \ldots & 0 & 0 & & 0
\end{array}\right)_{(m+1) \times(m+1)}
\end{aligned}
$$


and $h_{i}=\lambda_{i} h$ for $i=1,2,3, \ldots, m+1$, where $h$ is in $H$ with $\|h\|=1$, then

(*) $\left\langle P_{m+1}^{c}\left(\begin{array}{c}h_{1} \\ h_{2} \\ \vdots \\ h_{m+1}\end{array}\right),\left(\begin{array}{c}h_{1} \\ h_{2} \\ \vdots \\ h_{m+1}\end{array}\right)\right\rangle=2+2 \operatorname{Re}\left(c\left\langle S_{m+1} \lambda, \lambda\right\rangle\langle T h, h\rangle\right) \geq 0$.

Similarly,

$$
\text { (**) } \quad 2-2 \operatorname{Re}\left(c\left\langle S_{m+1} \lambda, \lambda\right\rangle\langle T h, h\rangle\right) \geq 0 .
$$

From $(*)$ and $(* *)$, we have $\left|\operatorname{Re} c\left\langle S_{m+1} \lambda, \lambda\right\rangle\langle T h, h\rangle\right| \leq 1$ for all $|c|=1$. Hence, $\left|\left\langle S_{m+1} \lambda, \lambda\right\rangle\langle T h, h\rangle\right| \leq 1$ or

$$
w(T) \leq 1 / w\left(S_{m+1}\right)=1 / \cos \frac{\pi}{m+2} \quad[1, \text { Proposition 1] }
$$

Therefore, (3) implies (1).

\section{Proposition 2.2.}

$$
\begin{aligned}
\min \left\{\rho:\left(\begin{array}{cccccc}
\rho & & T & 0 & \ldots & 0 \\
& & & & & \vdots \\
T^{*} & & \rho & T & & 0 \\
0 & & & & & \\
\vdots & & & & \ddots & T \\
0 & \ldots & 0 & & T^{*} & \rho
\end{array}\right)_{n \times n} \geq 0 \begin{array}{l}
\text { for nonnegative } \\
\text { number } \rho \text { and for all } n
\end{array}\right\} \\
\quad=2 w(T) .
\end{aligned}
$$

Proof. Suppose that

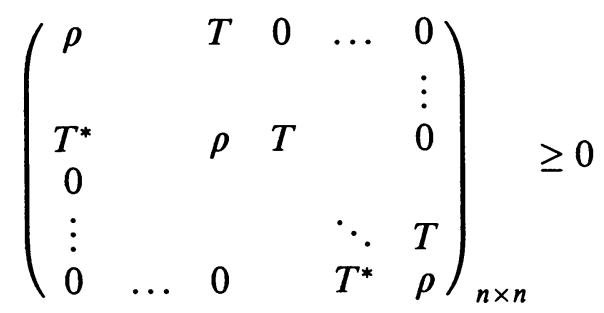

for some $\rho \geq 0$ and for all $n$. Applying Proposition 2.1, we have $w(2 T / \rho) \leq 1$ or $w(2 T) \leq \rho$. Since $w(T / w(T))=1$, we have

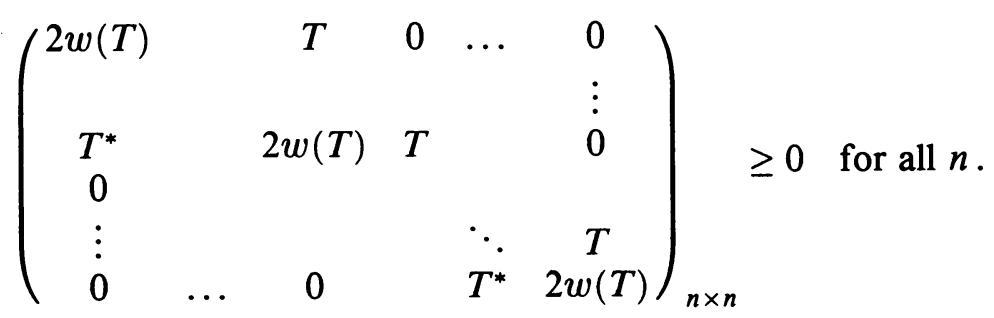


Corollary 2.3. If

$$
\left(\begin{array}{cccccc}
\rho & & T & 0 & \ldots & 0 \\
& & & & & \vdots \\
T^{*} & & \rho & T & & 0 \\
0 & & & & & \\
\vdots & & & & \ddots & T \\
0 & \ldots & 0 & & T^{*} & \rho
\end{array}\right)_{n \times n} \geq 0
$$

and $w(T)>\rho / 2$, then $n \leq\left[\pi / \cos ^{-1}\left(\frac{\rho}{2 w(T)}\right)-1\right]$, where $[x]$ is the greatest integer less than or equal to $x$. Moreover, $\max \left\{m: w(T) \leq \rho / 2 w\left(S_{m}\right)\right\}=$ $\left[\pi / \cos ^{-1}\left(\frac{\rho}{2 w(T)}\right)-1\right]$.

Proof. Since

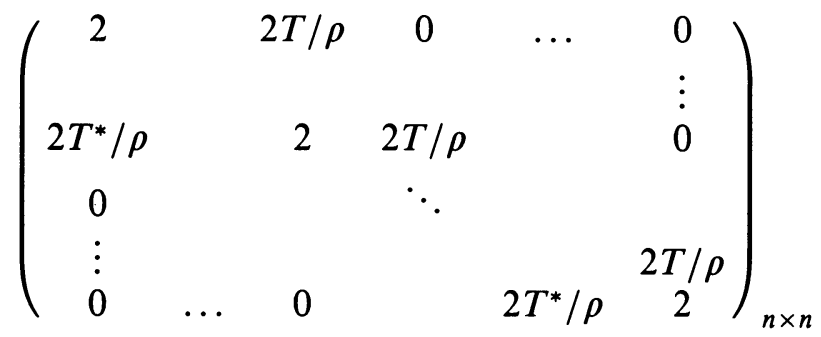

is positive, from the proof of Proposition 2.1, we have

$$
w\left(\frac{2 T}{\rho}\right) \leq \frac{1}{w\left(S_{n}\right)}=\frac{1}{\cos (\pi /(n+1))} .
$$

Let $m_{0}=\max \left\{m: w(2 T / \rho) \leq 1 / w\left(S_{m}\right)\right\}$; then $w(2 T / \rho) \leq 1 / w\left(S_{m_{0}}\right)$. Hence $n \leq m_{0} \leq \pi / \cos ^{-1}\left(\frac{\rho}{2 w(T)}\right)-1$. Now, $w(2 T / \rho)>1 / w\left(S_{m_{0}+1}\right)$, we have $\pi / \cos ^{-1}\left(\frac{\rho}{2 w(T)}\right)-2<m_{0}$. Therefore, $m_{0}=\left[\pi / \cos ^{-1}\left(\frac{\rho}{2 w(T)}\right)-1\right]$, where $[x]$ is the greatest integer less than or equal to $x$.

Example 2.4. From [1, Proposition 1] and Proposition 2.2, we have

$$
\begin{aligned}
& \min \left\{\rho:\left(\begin{array}{cccccc}
\rho & & S_{m+1} & 0 & \cdots & 0 \\
& & & & & \vdots \\
S_{m+1}^{*} & & \rho & S_{m+1} & & 0 \\
0 & & & \ddots & & \\
\vdots & & & & & S_{m+1} \\
0 & \ldots & 0 & & S_{m+1}^{*} & \rho
\end{array}\right)_{n \times n} \geq 0 \text { for all } n\right\} \\
& =2 w\left(S_{m+1}\right)=2 \cos \frac{\pi}{m+2}
\end{aligned}
$$

and $\left\|S_{m}\right\|=1$. Let

$$
x=\frac{1}{\sqrt{m+1}}\left(\begin{array}{c}
1 \\
1 \\
\vdots \\
1
\end{array}\right)
$$

then

$$
w\left(S_{m+1}\right)=\cos \frac{\pi}{m+2} \geq\left\langle S_{m+1} x, x\right\rangle=\frac{m}{m+1} .
$$


If $m>1$, then $2 w\left(S_{m+1}\right) \geq \frac{2 m}{m+1}>1$. Hence,

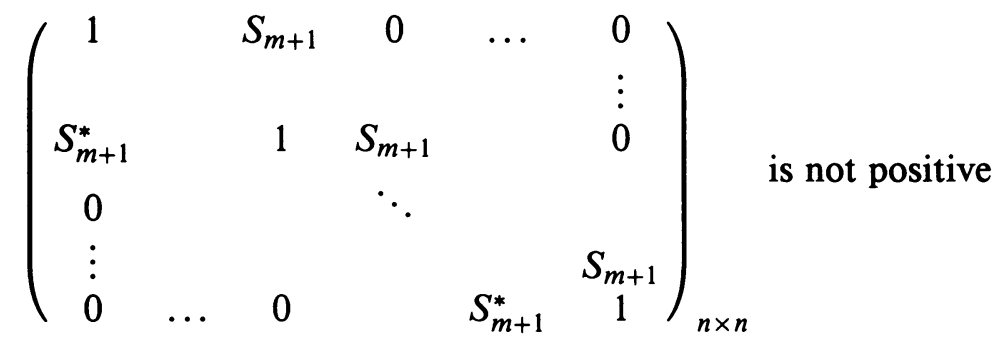

when $n>\pi / \cos ^{-1}\left(1 / 2 w\left(S_{m+1}\right)\right)-1$.

Corollary 2.5. If

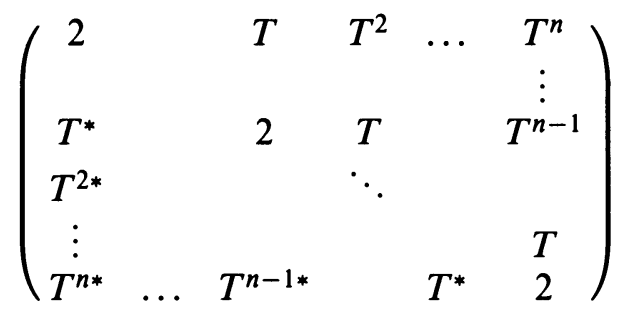

is positive and $w(T)>1$, then $n \leq 1\left[\pi / \cos ^{-1}\left(\frac{1}{2 w(T)}\right)-1\right]$, where $[x]$ is the greatest integer less than or equal to $x$.

Proof. From the proof of Proposition 2.1, we have

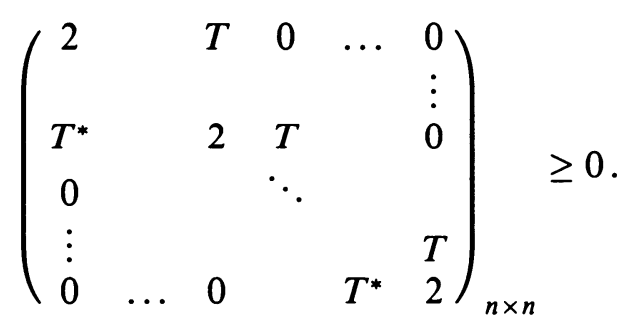

Applying Corollary 2.3, we have the corollary.

Theorem 2.6 [4, Theorem 2.7]. Let $L: A \rightarrow B(H)$ be a completely bounded linear map. Then

$$
\begin{aligned}
S(L) & =\min \left\{\|\phi\|_{\mathrm{cb}}: \phi \pm \operatorname{Re} c L \text { is completely positive for all }|c|=1\right\} \\
& =\min \left\{w(T): L \text { has a minimal commutant representation } V^{*} T \Pi V\right\} .
\end{aligned}
$$

Theorem 2.7.

$$
\min \left\{\|\phi\|_{\mathrm{cb}}:\left(\begin{array}{cccccc}
\phi & & L & 0 & \ldots & 0 \\
& & & & & \vdots \\
L^{*} & & \phi & L & & 0 \\
0 & & & \ddots & & \\
\vdots & & & & & L \\
0 & \ldots & 0 & & L^{*} & \phi
\end{array}\right)_{n \times n} \begin{array}{l}
\text { is completely } \\
\text { positive for all } n
\end{array}\right\}=2 S(L)
$$


Proof. If

$$
\left(\begin{array}{cccccc}
\phi & & L & 0 & \ldots & 0 \\
& & & & & \vdots \\
L^{*} & & \phi & L & & 0 \\
0 & & & \ddots & & \\
\vdots & & & & & L \\
0 & \ldots & 0 & & L^{*} & \phi
\end{array}\right)_{n \times n}
$$

is completely positive for some $\phi$ and for all $n$, then there exists a completely positive map $\psi$ with $\psi(I)=\|\phi\|_{\mathrm{cb}} I$ such that

$$
\left(\begin{array}{cccccc}
\psi & & L & 0 & \ldots & 0 \\
& & & & & \vdots \\
L^{*} & & \psi & L & & 0 \\
0 & & & \ddots & & \\
\vdots & & & & & L \\
0 & \ldots & 0 & & L^{*} & \psi
\end{array}\right)_{n \times n}
$$

is completely positive. Since $\left(\begin{array}{cc}\psi & L \\ L^{*} & \psi\end{array}\right)$ is completely positive, applying [3, Theorem 2.2], we have $\psi=\|\phi\|_{\mathrm{cb}} V^{*} \sqcap V$ and $L=\|\phi\|_{\mathrm{cb}} V^{*} T \sqcap V$, where $V$ is an isometry, $\Pi$ is a $*$-representation, and $T$ is in $\Pi(A)^{\prime}$. Applying [3, Proposition 2.6], we have

$$
\|\phi\|_{\mathrm{cb}}\left(\begin{array}{cccccc}
1 & & T & 0 & \ldots & 0 \\
& & & & & \vdots \\
T^{*} & & 1 & T & & 0 \\
0 & & & \ddots & & \\
\vdots & & & & & T \\
0 & \ldots & 0 & & T^{*} & 1
\end{array}\right)_{n \times n} \geq 0
$$

Applying Proposition 2.2, we have $2 w(T) \leq 1$. Using Theorem 2.6, we have

$$
2 S(L) \leq 2 w\left(\|\phi\|_{\mathrm{cb}} T\right) \leq\|\phi\|_{\mathrm{cb}} .
$$

Conversely, applying [4, Theorem 2.7], there exist an isometry $\bar{V}$, a *-representation $\bar{\Pi}$, and a bounded linear operator $\bar{T}$ in $\bar{\Pi}(A)^{\prime}$ such that $L=\bar{V}^{*} \bar{T} \bar{\Pi} \bar{V}$ with $w(\bar{T})=S(L)$. Since

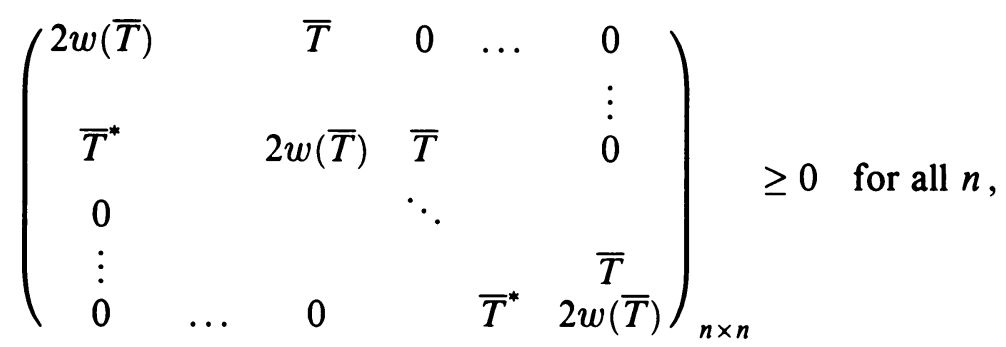


applying [3, Proposition 2.6], we have that

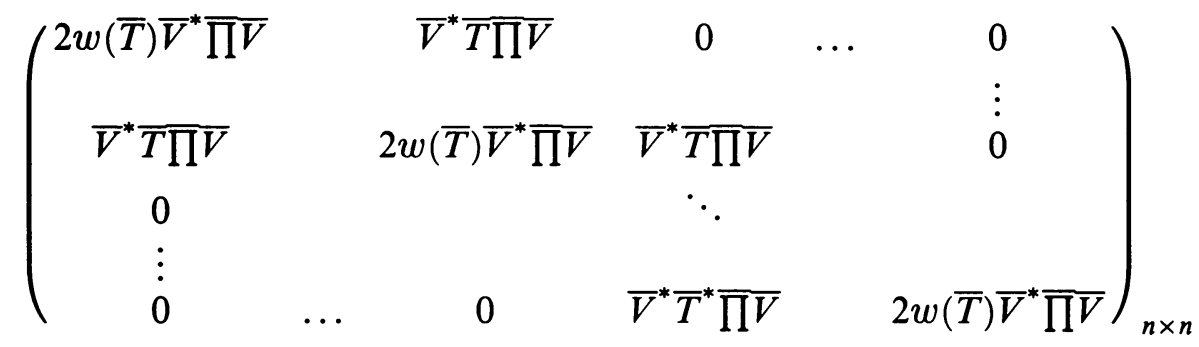

is completely positive. Hence we have proved the theorem.

Example 2.8. Let $L: C \oplus C \rightarrow M_{2}(C)$ be defined by

$$
a \oplus b \rightarrow \frac{1}{\sqrt{2}}\left(\begin{array}{ll}
a & 0 \\
b & 0
\end{array}\right)
$$

Let the completely positive map $\psi: C \oplus C \rightarrow M_{2}(C)$ be defined by

$$
a \oplus b \rightarrow \frac{1}{\sqrt{2}}\left(\begin{array}{cc}
a+b & 0 \\
0 & a+b
\end{array}\right) \text {. }
$$

It is not difficult to see that $\left(\begin{array}{cc}\psi & L \\ L^{*} & \psi\end{array}\right)$ is completely positive and

$$
\|\psi\|_{\mathrm{cb}}=\sqrt{2}=\min \left\{\|\phi\|_{\mathrm{cb}}:\left(\begin{array}{cc}
\phi & L \\
L^{*} & \phi
\end{array}\right) \text { is completely positive }\right\} \text {. }
$$

Since $\|L\|_{\mathrm{cb}}=1$ and $S(L)=(1+\sqrt{2}) / 2 \sqrt{2}$ [4, Example 2.8], we have

$$
\begin{aligned}
& S(L)<\|L\|_{\mathrm{cb}}<\|\psi\|_{\mathrm{cb}}<2 S(L) \\
& =\min \left\{\|\phi\|_{\mathrm{cb}}:\left(\begin{array}{cccccc}
\phi & & L & 0 & \ldots & 0 \\
& & & & & \vdots \\
L^{*} & & \phi & L & & 0 \\
0 & & & \ddots & & \\
\vdots & & & & & L \\
0 & \ldots & 0 & & L^{*} & \phi
\end{array}\right)_{n \times n} \text { positive for all } n\right\} \text {. }
\end{aligned}
$$

Corollary 2.9. If $L$ is a self-adjoint completely bounded linear map, then

$$
2\|L\|_{\mathrm{cb}}=\min \left\{\|\phi\|_{\mathrm{cb}}:\left(\begin{array}{cccccc}
\phi & & L & 0 & \ldots & 0 \\
& & & & & \vdots \\
L & & \phi & L & & 0 \\
0 & & & \ddots & & \\
\vdots & & & & & L \\
0 & \ldots & 0 & & L & \phi
\end{array}\right)_{n \times n} \begin{array}{l}
\text { is completely } \\
\text { positive for all } n
\end{array}\right. \text {. }
$$

Proof. Applying [4, Corollary 2.9], we have $S(L)=\|L\|_{\mathrm{cb}}$. 
Corollary 2.10. If $L$ is a bounded linear functional, then

$$
2\|L\|=\min \left\{\|\phi\|_{\mathrm{cb}}:\left(\begin{array}{cccccc}
\phi & & L & 0 & \ldots & 0 \\
& & & & & \vdots \\
L^{*} & & \phi & L & & 0 \\
0 & & & \ddots & & \\
\vdots & & & & & L \\
0 & \ldots & 0 & & L^{*} & \phi
\end{array}\right)_{n \times n} \begin{array}{l}
\text { is completely } \\
\text { positive for all } n
\end{array}\right\} .
$$

Proof. Since $\|L\|=S(L)$ [4, Corollary 3.3], we have the corollary.

Corollary 2.11. If

$$
\left(\begin{array}{cccccc}
\phi & & L & 0 & \ldots & 0 \\
& & & & & \vdots \\
L^{*} & & \phi & L & & 0 \\
0 & & & \ddots & & \\
\vdots & & & & & L \\
0 & \ldots & 0 & & L^{*} & \phi
\end{array}\right)_{n \times n}
$$

is completely positive with $n>1$ and $0<\|\phi\|_{\mathrm{cb}}<2 S(L)$, then

$$
n \leq\left[\frac{\pi}{\cos ^{-1}\left(\|\phi\|_{\mathrm{cb}} / 2 S(L)\right)}-1\right] \text {, }
$$

where $[x]$ is the greatest integer less than or equal to $x$.

Proof. From the proof of the Theorem 2.7, we have

$$
w\left(\|\phi\|_{\mathrm{cb}} T\right) \geq S(L)>\frac{1}{2}\|\phi\|_{\mathrm{cb}} \text { and }\left(\begin{array}{cccccc}
1 & & T^{*} & 0 & \ldots & 0 \\
& & & & & \vdots \\
T^{*} & & 1 & T & & 0 \\
0 & & & \ddots & & \\
\vdots & & & & & T \\
0 & \ldots & 0 & & T^{*} & 1
\end{array}\right)_{n \times n} \geq 0 .
$$

Since $w(T)>1 / 2$ and $1 / 2 w(T) \leq\|\phi\|_{\mathrm{cb}} / 2 S(L)<1$, applying Corollary 2.3, we obtain

$$
n \leq\left[\frac{\pi}{\cos ^{-1}(1 / 2 w(T))}-1\right] \leq\left[\frac{\pi}{\cos ^{-1}\left(\|\phi\|_{\mathrm{cb}} / 2 S(L)\right)}-1\right] .
$$

Example 2.12. If $\psi$ and $L$ are from Example 2.8 and

$$
\left(\begin{array}{cccccc}
\psi & & L & 0 & \ldots & 0 \\
& & & & & \vdots \\
L^{*} & & \psi & L & & 0 \\
0 & & & \ddots & & \\
\vdots & & & & & L \\
0 & \ldots & 0 & & L^{*} & \psi
\end{array}\right)_{n \times n}
$$


is completely positive, then

$$
n \leq\left[\frac{\pi}{\left.\cos ^{-1}(2 / 1+\sqrt{2})\right)}-1\right]=4 .
$$

\section{ACKNOWLEDGMENT}

The author gratefully acknowledges several valuable conversations with Professor Vern Paulsen.

\section{REFERENCES}

1. U. Haagerup and P. de la Harpe, The numerical radius of a nilpotent operator on a Hilbert space, Proc. Amer. Math. Soc. 115 (1992), 371-379.

2. V. I. Paulsen, Completely bounded maps and dilations, Pitman Res. Notes Math. Ser., vol. 146, Longman Sci. Tech., London, 1986.

3. V. I. Paulsen and C. Y. Suen, Commutant representations of completely bounded maps, J. Operator Theory 13 (1985), 87-101.

4. C. Y. Suen, The numerical radius of a completely bounded map, Acta Math. Hungar. 59 (1992), 283-289.

Department of General Academics, Texas A \& M University at Galveston, P. O. Box 1675, Galveston, TeXas 77553-1675

E-mail address: suen_cetamug.tamu.edu 